\title{
Erratum
}

\section{Bethe-Salpeter Equation with Confining Kernel; Correct Non-Relativistic Limit and the Constant to be Added to the Linear Potential}

Dieter Gromes

Z. Phys. C - Particles and Fields 11, 147 (1981)

In (2.4) replace $2 v$ by $2^{v}$ in the denominator on the right-hand side.

In the equation following (2.20) add $-(2 \pi)^{3} \lambda \mathbf{r}_{0} \delta^{(3)}(\mathbf{q})$ to the right-hand side. 